\title{
Drinking habits as cofactors of risk for alcohol induced liver damage
}

S Bellentani, G Saccoccio, G Costa, C Tiribelli, F Manenti, M Sodde, L Saveria Croce', F Sasso, G Pozzato, G Cristianini, G Brandi and the Dionysos Study Group

\begin{abstract}
Background-The Dionysos Study is a cohort study of the prevalence of chronic liver disease in the general population of two northern Italian communities. It included 6917 subjects, aged $12-65(69 \%$ of the total population).
\end{abstract}

Aims-The aim of this part of the study was to examine the relationship of daily alcohol intake, type of alcoholic beverage consumed, and drinking patterns to the presence of alcohol induced liver damage in an open population.

Patients and methods-6534 subjects, free of virus related chronic liver disease and participating in the first cross-sectional part of the study, were fully examined. Each subject underwent: (a) medical history and physical examination, (b) evaluation of alcohol intake using an illustrated dietary questionnaire, and (c) routine blood tests. More invasive diagnostic procedures were performed when indicated. Results-Multivariate analysis showed that the risk threshold for developing either cirrhosis or non-cirrhotic liver damage (NCLD) was ingestion of more than $30 \mathrm{~g}$ alcohol per day in both sexes. Using this definition, 1349 individuals (21\% of the population studied) were at risk. Of these, only $74 \quad(5.5 \%$ of the individuals at risk) showed signs of liver damage. The prevalence of "pure" alcoholic cirrhosis was $0.43 \%$ (30 of 6917), representing $2.2 \%$ of the individuals at risk, with a ratio of men to women of 9:1, while $44(3.3 \%$ of the individuals at risk) showed persistent signs of NCLD. After 50 years of age, the cumulative risk of developing both NCLD and cirrhosis was significantly higher $(p<0.0001)$ for those individuals who regularly drank alcohol both with and without food than for those who drank only at mealtimes.

Conclusions-Our data show that in an open population the risk threshold for developing cirrhosis and NCLD is $30 \mathrm{~g}$ ethanol/day, and this risk increases with increasing daily intake. Drinking alcohol outside mealtimes and drinking multiple different alcoholic beverages both increase the risk of developing alcohol induced liver damage.

(Gut 1997; 41: 845-850)

Keywords: alcohol; liver disease; cirrhosis
Alcohol abuse is considered a major cause of both acute and chronic liver disease. ${ }^{1}$ In many retrospective studies a close relationship between cumulative alcohol intake and risk of developing liver damage have been observed. ${ }^{1-10}$ However, data based on prospective studies ${ }^{11-14}$ are controversial, and pertinent data derived from epidemiological cohort studies based on open populations are not available to confirm this widely held belief. Moreover, it is not completely clear whether alcohol induced chronic liver disease is related solely to the total amount of alcohol ingested over time or whether other factors, such as genetic factors, type of alcoholic beverage ingested, and drinking patterns, play a significant role..$^{15-19}$

The Dionysos Study is the first cohort study to explore the prevalence of chronic liver disease in an entire adult population. Some data from the Dionysos Study have already been published ${ }^{20}$ and show that the prevalence of chronic liver disease is at least twice that previously reported for selected populations from the industrialised world. As one would expect, viral infection (especially hepatitis C) and alcohol abuse are the most significant causes. Moreover, excessive alcohol intake plays a major role in the deterioration of virus related chronic liver disease and its progression to hepatocellular carcinoma (HCC) ${ }^{20}$

In the present paper we explore in greater detail the relationships between daily amount of alcohol ingested by the participants in the Dionysos Study, type of alcoholic beverage consumed, drinking patterns (only at mealtimes or both with and without food) and the development of cirrhosis or non-cirrhotic chronic liver damage (NCLD) as well as HCC.

\section{Patients and Methods}

The details of the overall design of the Dionysos Study have already been published. ${ }^{20}$ Briefly all the 10151 inhabitants, ranging in age between 12 and 65, of two northern Italian communities were considered eligible for the study. In each patient, as described more fully elsewhere, ${ }^{20}$ the following were carried out. (1) An extensive medical history was obtained including previous diagnosis of chronic liver disease. (2) Alcohol intake was evaluated using a semiquantitative colour-illustrated food questionnaire $^{2122}$ including detailed questions on the use of alcoholic beverages. ${ }^{23}{ }^{24}$ The questionnaire was administered to the enrolee by a medical staff member, who specialised in either gastroenterology or nutrition. All the
Accepted for publication 24 June 1997 
medical personnel were trained in a full immersion three-day course before the beginning of the study in order to be able to administer the questionnaire uniformly. ${ }^{20}$ The subjects were asked in multiple choice form whether they drank beer, red wine, white wine, alcoholic aperitifs, or hard liquors "daily", "weekly", "monthly", or "hardly ever/never". For each type of alcoholic beverage, a colourillustrated picture of the glass containing a standard unit of the beverage $(200 \mathrm{ml}$ for beer, $100 \mathrm{ml}$ for wine, $70 \mathrm{ml}$ for aperitif, and $40 \mathrm{ml}$ for hard liquor) was printed in the questionnaire. All subjects were also asked if they usually drank a glass like the one reported in the picture, or a smaller or a larger one. When a smaller or larger glass than the one reproduced in the picture was used, the amount of the beverage per day was either reduced or increased by $25 \%$. In order to calculate exactly the amount of alcohol in grams for each unit (glass), the brand of beverage was also recorded. The duration of use and the time of drinking (morning, afternoon or night) of each type of alcoholic beverage was also recorded. The questionnaire also contained the question: "Do you drink only at mealtimes or also without food?" Daily alcohol intake (in grams) was computed by multiplying the frequency of consumption of each unit of beverage by the alcohol content of the specified portions, according to standard procedures. ${ }^{21-24}$ The total amount of alcohol consumed in a lifetime (in $\mathrm{kg}$ ) was computed by multiplying the daily alcohol intake (in grams) by the duration of intake (in years). Alcohol consumption evaluated using the semiquantitative questionnaire was also validated by cross-checking with family members. (3) A detailed physical examination was performed to detect hepatobiliary diseases or physical signs related to chronic liver disease; the body mass index (BMI) was also recorded. (4) Blood samples were taken to check serum alanine aminotransferase, serum aspartate aminotransferase, $\gamma$-glutamyl transferase (GGT), mean cell volume and platelet count. Hepatitis B surface antigen (HBsAg), anti-HBsAg (diagnostic kits from Abbott Laboratories, North Chicago, IL, USA) and anti-hepatitis $\mathrm{C}$ virus (HCV) (enzyme linked immunosorbent assay (ELISA), second and third generation; Ortho Diagnostic Systems, Raritan, NJ, USA) were also screened.

CRITERIA FOR CLASSIFICATION OF CIRRHOSIS AND NCLD

Each participant who on initial examination had at least one abnormal blood test or physical sign suggestive of liver damage was classified as having suspected liver disease. These patients underwent the following additional procedures.

(1) Repetition of the same blood tests plus other "liver function" tests, including: serum alkaline phosphatase, bilirubin, albumin, $\gamma$-globulin, prothrombin time; blood assay of glucose, cholesterol, triglycerides. The complete set of hepatitis $\mathrm{B}$ virus markers (anti-HBc, $\mathrm{HBeAg}$, anti-
$\mathrm{HBe}$ ) and hepatitis delta virus (Abbot Diagnostics ELISA kit, North Chicago, IL, USA) was tested if the subject was hepatitis $B$ virus positive.

(2) Ultrasonography of the liver, biliary system, pancreas, and spleen, with measurement of portal vein and retropancreatic splenic vein diameters. Ultrasonography was always performed by the same operator.

(3) Other more complex and invasive diagnostic procedures such as liver computed tomography scan and percutaneous liver biopsy, when indicated.

The diagnosis of cirrhosis was clinically suspected when at least two of the following features were present:

(a) spider nevi, scleral icterus, palmar erythema, ascites, flapping tremor, hepatic or spleen enlargement;

(b) platelet count less than 140 000;

(c) portal vein diameter greater than $12 \mathrm{~mm}$ or irregular margins of the liver parenchyma at ultrasonography.

The diagnosis of cirrhosis and HCC was confirmed by liver biopsy in all the 35 cases where the diagnosis was suspected clinically.

All the subjects without liver cirrhosis as defined above and with persistent levels of alanine aminotransferase, aspartate aminotransferase, or GGT twice the upper normal range in at least two consecutive one month interval checks and with signs of steatosis at ultrasonography were classified as having NCLD. Of these patients, $70 \%$ underwent liver biopsy, which confirmed the diagnosis of alcoholic hepatitis or steatosis. The remainder either refused biopsy or biopsy was not justified on ethical grounds. Subjects with positive viral hepatitis markers were excluded.

\section{STATISTICAL ANALYSIS}

Statistical analysis was performed with an SPSS/PC statistical package (SPSSinc., Chicago, IL, USA). All $\mathrm{p}$ values reported are twotailed. Statistical comparison between means was calculated with the one-way analysis of variance, and when the variances were not homogeneous, with the Kruskal-Wallis oneway analysis of variance. ${ }^{25}$ The association between either the presence of cirrhosis and HCC or NCLD and all the categorical variables (including the continuous variables, which were analysed with cut-off values) were assessed with the unadjusted $\chi^{2}$ statistics. ${ }^{25}$ The variables included in the analysis were: alcohol intake expressed in different ways ( $\mathrm{g} /$ day or $\mathrm{kg} /$ lifetime, both corrected and not corrected for BMI), the types of alcoholic beverage consumed, and drinking pattern (only at mealtimes or with and without food). A logisticregression model $^{26}$ was used in the multivariate modelling of associations. All the factors for which the $p$ value of univariate and discriminant analysis was less than 0.05 were entered in the model, but the factors with the highest $\mathrm{p}$ values on multivariate analysis were dropped sequentially until all the factors in the model had $p$ values of less than 0.05 . The analysis was also repeated after stratification for both sexes. 
TABLE 1 Classes of daily alcohol consumption of the screened population $(n=6534)$, expressed as absolute number ( $n$ ) and percentage of lines (\%), according to the presence of either non-cirrhotic alcohol-induced liver damage (NCLD) or cirrhosis or hepatocellular carcinoma (=cirrhosis)

\begin{tabular}{|c|c|c|c|c|c|}
\hline \multirow{2}{*}{$\begin{array}{l}\text { Alcohol intake } \\
\text { (g/day) }\end{array}$} & \multirow[b]{2}{*}{ No $(n=6442)$} & \multirow{2}{*}{$\begin{array}{l}N C L D \\
(n=57)\end{array}$} & \multirow{2}{*}{$\begin{array}{l}\text { Cirrhosis } \\
(n=35)\end{array}$} & \multicolumn{2}{|c|}{$\begin{array}{l}\text { Multivariate analysis (odds ratio) } \\
(95 \% \text { CI) }\end{array}$} \\
\hline & & & & For $N C L D^{a}$ & For cirrhosis $^{a}$ \\
\hline Teetotallers & $2501(99.9)$ & $0(0)$ & $1(0.04)$ & - & - \\
\hline $0.1-30$ & $2666(99.3)$ & $13(0.5)$ & $4(0.15)$ & $-^{\mathrm{b}}$ & $-^{\mathrm{b}}$ \\
\hline $31-60$ & $745(97.2)$ & $14(1.8)$ & $8(1.0)$ & 7.5 (3.5 to 15.9$)$ & $10.9(3.6$ to 33.5$)$ \\
\hline $61-90$ & $276(93.0)$ & $14(4.7)$ & $7(2.3)$ & $20.2(9.4$ to 43.3$)$ & $25.0(7.9$ to 79.3$)$ \\
\hline $91-120$ & $132(91.6)$ & $5(3.5)$ & $7(4.9)$ & 15.1 (5.3 to 42.8 ) & 52.9 (16.6 to 169$)$ \\
\hline$>120$ & $122(86.5)$ & $11(7.8)$ & $8(5.7)$ & 35.8 (15.7 to 81.6$)$ & $62.3(20.1$ to 193$)$ \\
\hline
\end{tabular}

Anti-HCV and HbsAg positive subjects ( $n=313$ ), and 70 subjects in whom either the food questionnaire or the blood tests were not completed were also excluded.

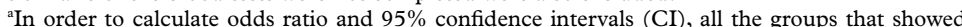
statistical significance for the presence of either NCLD or cirrhosis at the multivariate analysis (that is, people drinking more than $30 \mathrm{~g} /$ day) were compared with the teetotallers and the moderate drinkers $(<30 \mathrm{~g} /$ day $)$ grouped together. Above this threshold limit $\mathrm{p}$ was always less than 0.00001 .

${ }^{\mathrm{b}} \mathrm{p} v$ teetotallers, not significant.

Odds ratios (ORs) and $95 \%$ confidence intervals (CI) were also calculated. ${ }^{25}$ KaplanMeier survival and cumulative hazard risk curves for the presence of NCLD and cirrhosis according to age and stratified for the pattern and whether alcohol was consumed only with meals or at other times as well were also calculated. $^{27}$

\section{Results}

STUDY POPULATION, COMPLIANCE, AND

ESTIMATION OF BIAS IN PATIENT ACCESSION

During the two years of the study, of the 10151 people who were contacted, 6917 were enrolled $($ compliance $=69 \%)$. Those citizens who did not respond to the first invitation were reinvited once, either by letter or by telephone call, but the percentage of accession of this group was low (15\%). As previously reported, ${ }^{20}$ overall compliance was higher in women than in men (73 v66\%; difference not significant). Young people, especially those aged 18-35, showed lower compliance than older people (65\% $v 77 \%$ difference not significant).

The results reported in this paper are based on 6534 subjects - that is, the entire original cohort $(n=6917)$, from which anti-HCV and HBsAg positive subjects $(\mathrm{n}=314)$ were removed. Seventy subjects who either failed to get their blood tests or complete the questionnaire were also removed.

In order to identify possible bias, we reviewed epidemiological data from 1991 on the entire population of the two provinces containing the Dionysos Study towns (about 1 million people) and compared them with demographic characteristics of the cohort enrolled. As described more fully elsewhere, ${ }^{20}$ the ratio of men to women, wine intake (litres/ year per person), and prevalence of gallstones were very similar in our study group and the broader population.

DAILY ALCOHOL CONSUMPTION AND ETHANOL INDUCED LIVER DAMAGE

Of the 6534 subjects studied, 327 (5\%) showed only minimal and sporadic biochemical alterations, mainly of GGT (41\%) or both alanine aminotransferase and GGT (59\%), and were considered to be healthy. Multiple logistic regression analysis of the data showed that the risk of having either cirrhosis or NCLD, defined as in the Patients and Methods section, was significant with a lifetime alcohol intake higher than $100 \mathrm{~kg}$ and a daily alcohol intake higher than $30 \mathrm{~g}$ (table 1). Multivariate analysis with ORs are given in table 1. Men consumed more alcohol than women (43.8 (1) v 15.7 (0.4) g/day, mean (SEM); $\mathrm{p}<0.0001$ ), but the mean duration of drinking was similar in both sexes (21 (14) years for men and 20 (14) for women, mean (SD)). Above $30 \mathrm{~g} /$ day, the OR for the presence of NCLD and cirrhosis increased proportionally with daily alcohol consumption. The highest OR (35.8 and 62.3 respectively; $\mathrm{p}<0.0001$ ) occurred when daily consumption exceeded $120 \mathrm{~g} /$ day. Even at this highest level of alcohol intake, however, the percentage of subjects with alcohol induced liver damage was relatively low (19 of 141 $(13.5 \%))$.

If one assumes that an intake of $30 \mathrm{~g}$ alcohol per day is the risk threshold, 5185 of the 6534 subjects examined were not at risk for alcohol induced liver damage because they either drank less than $30 \mathrm{~g}$ alcohol/day $(\mathrm{n}=2683 ; 41 \%)$ or were teetotallers $(n=2501 ; 38.3 \%)$ (table 1$)$. Of these subjects not at risk, $13(0.25 \%)$ had NCLD, four $(0.08 \%)$ had cirrhosis, and one $(0.02 \%)$ had a capsulated primary liver cancer, which was surgically removed. Of the four cirrhotic patients, two were women with primary biliary cirrhosis, one was a man with haemochromatosis, and one was a man with insulin dependent diabetes with cryptogenic cirrhosis.

Of the remaining 1349 subjects at risk for alcohol induced liver damage because they drank $30 \mathrm{~g}$ or more alcohol per day, only 159 $(11.8 \%)$ were women. Two of the 159 women $(1.3 \%)$ and 42 of 1190 men $(3.6 \%)$ had NCLD, and three of 159 women $(1.9 \%)$ and 27 of 1190 men $(2.3 \%)$ had cirrhosis. Therefore the overall prevalence of alcoholic cirrhosis in the Dionysos cohort was $0.43 \%$. Two of the 27 cirrhotic men developed HCC during a follow up period of three years. The odds of having either NCLD or cirrhosis for drinkers who drank more than $30 \mathrm{~g}$ alcohol per day were 13.7 (range 7-25) and 23.6 (range 9-61) times those for drinkers who drank less. However, the positive predictive values for NCLD or cirrhosis of daily alcohol intake greater than $30 \mathrm{~g} /$ day was relatively low (3.3 and $2.2 \%$ respectively).

Multivariate analysis also showed that the effect of alcohol intake on disease risk is independent of the other variables examined, such as sex, body weight, BMI, and the single type of alcoholic beverage consumed, with the exclusion of the habits of drinking multiple drinks and of consuming alcohol both with and without food. After correction for BMI, as recently suggested by other authors, ${ }^{28}$ the calculated risk threshold was $0.9 \mathrm{~g}$ alcohol/BMI/day, and a sex difference in developing alcohol induced liver damage was found in the range of alcohol intake between 30 and $80 \mathrm{~g} /$ day. Within this range, the OR for the presence of cirrhosis and NCLD in women was twice as high as in men, but this difference, which resulted from the low number of women with alcohol induced liver 
TABLE 2 Average daily alcohol intake (g/day) (given as mean (SEM)) according to the different drinking habits (with meals only or at any time) and type of alcoholic beverage

\begin{tabular}{lll}
\hline & \multicolumn{2}{l}{ Alcohol consumed } \\
\cline { 2 - 3 } Type of beverage & With meals only $(n ; \%$ of lines) & At any time $(n ; \%$ of lines) \\
\hline Beer $(\mathrm{n}=102)$ & $5(1)(53 ; 52 \%)$ & $10(5)^{\star}(49 ; 48 \%)$ \\
Red wine only $(\mathrm{n}=205)$ & $12(1)(168 ; 82 \%)$ & $33(7)^{\star \star}(37 ; 18 \%)$ \\
White wine only $(\mathrm{n}=402)$ & $20(1)(375 ; 93 \%)$ & $60(9)^{\star \star} \dagger(27 ; 7 \%)$ \\
Hard liquors only $(\mathrm{n}=18)$ & $43(13)(8 ; 44 \%)$ & $11(2)^{\star}(10,56 \%)$ \\
Multiple drinks $(\mathrm{n}=3239)$ & $27(1)(1676 ; 52 \%)$ & $44(1)^{\star \star}(1563 ; 48 \%)$ \\
Total (n=3966) & $24(1)(2280 ; 57.5 \%)$ & $43(1)^{\star \star}(1686 ; 42.5 \%)$ \\
\hline
\end{tabular}

Teetotallers ( $n=2502)$, anti-HCV and HbsAg positive subjects $(n=313)$ and 136 subjects in whom information regarding type of beverage consumed was lacking were excluded from the analysis.

${ }^{\star} \mathrm{p}<0.05 v$ only with meals; ${ }^{\star \star} \mathrm{p}<0.01 v$ only with meals; $\nmid \mathrm{p}<0.05 v$ all beverage types consumed only with meals.
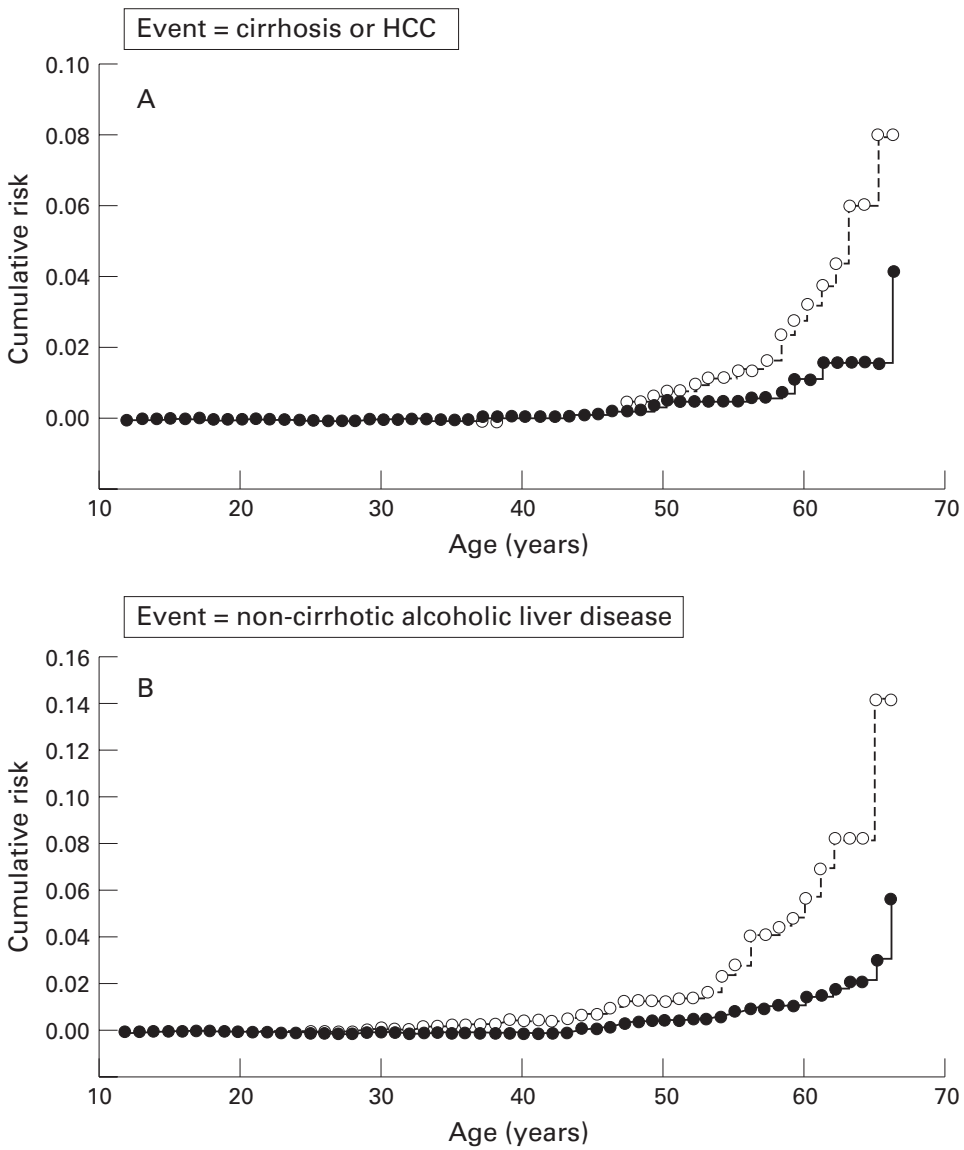

Figure 1: Cumulative hazard risk curves for the presence of non-cirrhotic alcohol induced liver disease (NCLD) (B) and cirrhosis or hepatocellular carcinoma $(A)$ as a function of age and drinking pattern in subjects with an alcohol intake greater than 30 g/day. Patients drinking only at mealtimes (•) were compared with those drinking outside mealtimes as well (O). The difference in risk of developing either NCLD or cirrhosis or HCC between people drinking alcohol only at mealtimes and those drinking outside mealtimes as well, calculated using log rank and Breslow tests, was significant $(p<0.001)$.

damage and the wide range of OR, did not reach statistical significance (data not shown).

DRINKING HABITS AND ALCOHOL INDUCED LIVER DAMAGE

In our population, monodrinkers of aperitifs were absent, while monodrinkers of beer were few $(\mathrm{n}=102)$ and drank less than $30 \mathrm{~g}$ alcohol/ day (7 (2), mean (SEM)). When the data on alcohol consumption were stratified according to drinking habits, it was evident that, except for the 18 hard liquor monodrinkers who consumed alcohol mainly at mealtimes, the daily alcohol intake was significantly higher when alcohol was consumed with and without food than only at mealtimes (43 (1) $v 24$ (1) g/day, mean (SEM); $\mathrm{p}<0.01$; table 2 ). This was true also for the 607 monodrinkers of wine, particularly those who drank only white wine (60 (9) $v 20$ (1) for white wine, and 33 (7) $v 12$ (1) for red wine; $\mathrm{p}<0.01$; table 2 ). The daily alcohol intake of white wine drinkers who drank ethanol also without food $(n=27)$ was significantly higher than that of all other categories of drinkers $(\mathrm{p}<0.05 v$ all categories, table 2). Drinkers who consumed more than one type of alcoholic beverage represented most of our population $(n=3239)$. The average daily alcohol intake of these subjects who also drank either with or without food (44 $(1), n=1563)$ was significantly higher than that of those who drank at mealtimes only $(27$ (1), $\mathrm{n}=1676 ; \mathrm{p}<0.01)$.

Multivariate analysis of the data showed that, independently of the amount ingested, drinking ethanol with and without food, and the use of multiple types of drinks were significantly associated with the presence of both NCLD and cirrhosis $(p<0.0001, p<0.0001$, and $\mathrm{p}<0.005$ respectively). The odds for having either cirrhosis or NCLD for drinkers who drank ethanol outside of mealtimes also was 3.4 (1.7-6.6) and 5.0 (2.9-8.5) times those for drinkers who drank only at mealtimes. Similarly, drinkers of multiple types of alcoholic beverages had a risk 23.2 (3-170) times higher for having cirrhosis than that of all other categories of drinkers.

Finally, we calculated cumulative hazard risk curves for having NCLD and cirrhosis in relation to age. The drinkers who drank $30 \mathrm{~g}$ ethanol/day or more and also drank both with and outside of meals had an increased prevalence of NCLD, and the increased risk began at 45 years. Similarly the risk for having cirrhosis began to increase at age 50 (fig 1).

\section{Discussion}

Alcohol abuse has been claimed as one of the most important causes of liver cirrhosis. ${ }^{12}{ }^{14}$ In Italy, alcohol has been reported to cause $25-65 \%$ of cirrhosis. ${ }^{2029} 30$ Most previous studies on the epidemiology of alcoholic cirrhosis were based on either selected or historical series. ${ }^{1-10}$ Furthermore no data were previously available that allowed a correct estimation of the prevalence of cirrhosis ascribed to the three main causes of these diseases (HBV, $\mathrm{HCV}$, and alcohol), alone or in combination. We found that $30 \mathrm{~g}$ alcohol/day is the minimal quantity of alcohol compatible with a measurable risk of developing cirrhosis for both sexes (table 1). By considering that this is the risk threshold, and that the overall prevalence of cirrhosis in the Dionysos study was $1.1 \%$ (78 over 6917$){ }^{20}$ we can calculate that the prevalence of "pure" alcoholic cirrhosis in the general population, at least of Northern Italy, is $0.43 \%$, and that alcohol is the sole cause of cirrhosis in $38 \%$ of the cases, with a ratio of men to women of 9:1. These data on the prevalence of alcohol induced liver disease in an entire stable adult population are unique. They are similar, however, to data from other epidemiological 
studies on the prevalence of alcohol related liver disease in broad adult populations. ${ }^{714}$

We also found a dose-effect relation between alcohol intake and alcohol induced hepatic damage, as reported by others. ${ }^{81314}$ We were not, however, able to confirm a significant sex difference in the minimum alcohol consumption necessary to increase the risk of having alcohol induced liver damage. Nor could we document a greater susceptibility for alcoholic liver damage in women than in men. Only in the range of alcohol intake between 30 and 80 $\mathrm{g} /$ day was a trend to a greater risk of developing alcohol induced liver disease in women than in men evident. Previous studies have suggested that women are more sensitive to alcohol than men. ${ }^{41431}$ Most studies, however, are retrospective studies and do not consider the distribution of alcohol intake in the population. In other prospective studies, a greater increase in relative risk of development of alcohol induced liver disease by increasing alcohol intake in women than in men was found. ${ }^{8-14}$ However, this could be due to a higher degree of underreporting of alcohol intake among women than men, since no sex differences in alcohol related risk of dying was found by the same authors in an earlier study. ${ }^{28}$ We believe that a greater hepatic susceptibility to damage by alcohol in women remains controversial. ${ }^{631}$ As we reported previously, the per capita wine consumption calculated from our dietary questionnaire was identical with that published by the Italian National Institute of Statistics for the general population in the years when the study was performed. ${ }^{20}$ Therefore a bias due to either an under- or an over-reporting of alcohol intake in our data seems unlikely. By dividing our population according to risk threshold we calculated that the odds for excessive drinkers (> $30 \mathrm{~g}$ alcohol/day) to develop persistent damage to their liver increased progressively with the amount of alcohol intake. However, the positive predictive value of daily alcohol intake for the presence of either NCLD or cirrhosis was relatively low (3.3 and $2.2 \%$ respectively). Therefore alcohol intake per se does not appear to be the sole determinant of liver damage in most individuals at risk. Genetic factors, as recently suggested, ${ }^{15-19}$ may be involved. Other factors, such as the type and pattern of alcohol ingested, may also be important. Our data demonstrate for the first time that consumption of alcohol either with or without meals and consumption of multiple types of alcoholic beverages are risk factors, independent of total consumption, for the presence of alcohol related liver disease. Drinking without food might differentially affect the intragastric metabolism of ethanol, by decreasing gastric alcohol dehydrogenase and hepatic glutathione, and by accelerating gastric emptying, as it occurs in rats. ${ }^{32} 33$

From the present data we conclude that the minimum alcohol intake associated with a significant increase in the prevalence of alcohol related liver disease was $30 \mathrm{~g} /$ day for both sexes. This risk increases in a dose related relationship, with no sex differences. The prevalence of "pure" alcoholic cirrhosis in the general population in Northern Italy is $0.43 \%$. However, the most striking result was that not only the quantity of alcohol consumed, but also the pattern of drinking was an important determinant in the risk of having NCLD or cirrhosis. Drinking without food and/or drinking multiple types of alcoholic beverages, independent of the amount drunk, is associated with an increased prevalence of alcohol related liver disease.

Our data suggest the usefulness of a nationwide educational campaign aimed at reducing alcohol intake, addressed primarily at young men, and focused on avoiding drinking outside of mealtimes and consuming only one type of drink, preferentially one with low alcohol concentration.

This study was supported by grants from Telethon (grant no E.396) Assessorato Sanita' Regione Friuli Venezia Giulia, Assessorato Sanita'Regione Emilia Romagna, Banca Popolare dell'Emilia Romagna, Caffe' Hag, Ente Nazionale Interprofessionale per la Valorizzazione del Vino, Esaote Biomedica, Fondo per lo Studio delle Malattie del Fegato, Gruppo Hesperia Strutture Sanitarie Private. The Dionysos Study Group is composed ture Sanitarie Private. The Dionysos Study Group is composed
of the following other active participants: C Armocida, MD; C of the following other active participants: C Armocida, MD; C
De Martin, MD; I Ferretti, BD; G F Figlioli, MD; N Fratti, Me Martin, MD; I Ferretti, BD; G F Figlioli, MD; N Fratti, MD; A L Manenti, MD; P Marchegiano, MD; B Messori, MD;
S Passamonti, MD; C Poppi, MD; R Sassatelli, MD. We thank S Passamonti, MD; C Poppi, MD; R Sassatelli, MD. We thank
William Gerry Morgan Hardison for constructive suggestions William Gerry Morgan Hardison for constructive
and criticisms and for revision of the manuscript.

1 Klatskin G. Alcohol and its relation to liver damage. Gastroenterology 1961;41:443--51.

2 Lelbach WK. Cirrhosis in the alcoholic and its relation to the volume of alcohol abuse. Ann NY Acad Sci 1975;252 $85-105$.

3 Pequignot G, Tuyns AJ, Berta JL. Ascitic cirrhosis in relation to alcohol consumption. Int $\mathcal{F}$ Epidemiol 1978;7: $113-20$

4 Tuyns AJ, Pequignot G. Greater risk of ascitic cirrhosis in females in relation to alcohol consumption. Int $\mathcal{F}$ Epidemiol 1984;13:53-7.

5 Marbet UA, Bianchi L, Meury U, Stadler GA. Long-term histological evaluation of the natural history and prognostic factors of alcoholic liver disease. F Hepatol 1987;4:364-72.

6 Grant BF, Dufour MC, Harford TC. Epidemiology of alcoholic liver disease. Semin Liver Dis 1988;8:12-25.

7 Sorensen TIA. The relationship between alcohol consumption and risk of development of cirrhosis of the liver. Alcologia 1990;2:121-6.

8 Klatsky AL, Armstrong MA, Friedman GD. Alcohol and mortality. Ann Intern Med 1992;117:646-54.

9 Batey RG, Burns T, Benson RJ, Byth K. Alcohol consumption and the risk of cirrhosis. Med f Aust 1992;156:413-16.

10 Lieber CS. Alcohol and the liver: 1994 update. Gastroenterology 1994;106:1085-105.

11 Saunders JB, Walters JRF, Davies P, Paton A. A 20-year prospective study of cirrhosis. BMf 1981;282:263-6.

12 Sorensen TIA, Ohrlom M, Bentsen KD, Hoybye G, Eghoje $\mathrm{K}$, Christoffersen P. Prospective evaluation of alcohol abuse and alcoholic liver injury in men as predictors of development of cirrhosis. Lancet 1984;2:241 1-44.

13 Bouchier IAD, Hislop WS, Prescott RJ. A prospective study of alcoholic liver disease and mortality. F Hepatol 1992;16: $290-7$.

14 Becker U, Deis A, Sorensen TIA, Gronbaek M, BorchJohnsen K, Müller CE, et al. Prediction of liver disease by alcohol intake, sex, and age: a prospective population study. Hepatology 1996;23:1025-9.

15 Hrubec Z, Omenn GS. Evidence of genetic predisposition to alcoholic cirrhosis and psychosis: twin concordances for alcoholism and its biological endpoints by zygosity among male veterans. Alcohol Clin Exp Res 1981;5:207-15.

16 Devor EJ, Reich T, Cloninger CR. Genetics of alcoholism and related end-organ damage. Semin Liver Dis 1988;8:1-

17 Hall PDLM. Genetic and acquired factors that influence ndividual susceptibility to alcohol-associated liver disease. f Gastroenterol Hepatol 1992;7:417-26.

18 Day CP, James OWF, Bassendine MF. Alcohol dehydrogenase polymorphisms and predisposition to alcoholic cirrhosis. Hepatology 1993;18:230-1.

19 Chao Y, Young T, Chang W, Tang HS, Hsu C. An investigation of whether polymorphisms of cytochrome P4502E1 are genetic markers of susceptibility of alcoholic end-stage organ damage in a chinese population. Hepatology 1995;22: organ dam.

20 Bellentani S, Tiribelli C, Saccoccio G, Sodde M, Fratti N. De Martin C, Cristianini G and the Dionysos Study Group. Prevalence of chronic liver disease in the general population of Northern Italy: the Dionysos Study. Hepatology 1994;20:1442-9. 
21 Block G. Hartmann AM, Dresser CM. A data-based approach to diet questionnaire design and testing. $A m \mathcal{F}$ Epidemiol 1986;124:453-69.

22 Cubean J, Pequinot G. La technique du questionnaire alimentaire quantitatif utilise par la section nutrition de I'INSERM. Rev Epidemiol Sante Publique 1980;28:367-72.

23 Midanik L. The validity of self-reported alcohol consumption and alcohol problems: a literature review. British fournal of Addiction 1982;77:357-82.

24 Paton A, Saunders JB. ABC of alcohol. BMf 1981;283: $1248-50$.

25 Fleiss JL. Statistical methods for rates and proportion. New York: Wiley and Sons, 1981

26 Hosmer DW Jr, Lemeshow S. Applied logistic regression. New York: John Wiley, 1989.

27 Meier P, Beach M. Biostatistics for the clinician. In Chalmer TC, ed. Data analysis for clinical medicine. Rome Rome International University Press, 1988:7-29.

28 Groenbaek M, Deis A, Sorensen TIA, Becker U, Borch Johnsen K, Müller C, et al. Influence of sex, age, body mass index, and smoking on alcohol intake and mortality. $B M$ 1994;308:302-6.
29 Ascione A, Barcellona R, Benini G, Budillon G, Candelaresi MT, Cataldi V, et al. Patologia cronica del fegato in Italia. Inchiesta retrospettiva multicentrica. Rec Prog Med 1977; 63:447-70.

30 De Bac C, Stroffolini T, Gaeta GB, Taliani G, Giusti G. Pathogenic factors in cirrhosis with and without hepatocellular carcinoma: a multicenter Italian study. Hepatology 1994;20:1225-30.

31 Frezza M, Di Padova C, Pozzato G, Terpin M, Baraona E, Lieber CS. High blood alcohol levels in women: the role of decreased gastric alcohol dehydrogenase activity and firstpass metabolism. N Engl f Med 1990;322:95-9.

32 Battiston L, Moretti M, Tulissi P, Micheli L, Marchi P, Lunazzi G, Pozzato G. Hepatic glutathione determination fter ethanol administration in rat: evidence of the first-pass metabolism of ethanol. Life Sci 1995;56:241-8.

33 Shaw S, Jayatilleke E, Ross WA, Gordon ER, Lieber CS. Ethanol induced lipid peroxidation: potentiation by longterm alcohol feeding and attenuation by methionine. $\mathcal{f} \mathrm{Lab}$ Clin Med 1981;98:417-25. 